\title{
Electro-thermal modeling of a microbridge gas sensor
}

\author{
Ronald P. Manginell, James H. Smith, Antonio J. Ricco, Robert C. Hughes, Daniel J. Moreno ${ }^{\dagger}$
}

Sandia National Laboratories, Albuquerque, NM 87185-1080.

\author{
Robert J. Huber \\ CONF-970968--7 \\ Dept. of Electrical Engineering, University of Utah, Salt Lake City, UT, 84112.
}

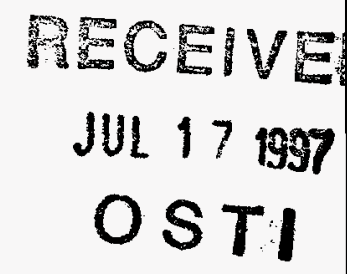

\begin{abstract}
Fully CMOS-compatible, surface-micromachined polysilicon microbridges have been designed, fabricated, and tested for use in catalytic, calorimetric gas sensing. To improve sensor behavior, extensive electro-thermal modeling efforts were undertaken using SPICE. The validity of the SPICE model was verified by comparing its simulated behavior with experimental results. The temperature distribution of an electrically-heated microbridge was measured using an infrared (IR) microscope. Comparisons among the measured distribution, the SPICE simulation, and distributions obtained by analytical methods show that heating at the ends of a microbridge has important implications for device response. Additional comparisons between measured and simulated current-voltage characteristics, as well as transient response characteristics, further support the accuracy of the model. A major benefit of electro-thermal modeling with SPICE is the ability to simultaneously simulate the behavior of a device and its control/sensing electronics. Results for the combination of a unique constant-resistance control circuit and microbridge gas sensor are given. Models of in situ techniques for monitoring catalyst deposition are shown to be in agreement with experiment. Finally, simulated chemical response of the detector is compared with the data, and methods of improving response through modifications in bridge geometry are predicted.
\end{abstract}

Key Words: Catalytic gas sensor, modeling, SPICE, $\mu$-CVD, in situ monitoring, CMOS, IR microscope

\section{INTRODUCTION}

1.1 Catalytic, calorimetric gas sensing: The electro-catalytic method

The lower explosive limit (LEL) is the concentration of gas in air below which it cannot be ignited. LEL has served as a practical concentration limit below which gas-air mixtures are considered free from explosions. ${ }^{1}$ For this reason most combustible gas detectors are used to detect concentrations up to the LEL and provide a direct measure of the flammability of a gas-air mixture. ${ }^{2}$ Combustible gas detection below the LEL is most often accomplished by catalytic, calorimetric means. ${ }^{3}$ The heat of oxidation of the combustible species on the surface of a hot catalyst is measured by means of a resistance thermometer in proximity with the catalyst. This method is, therefore, calorimetric. The heated catalyst permits oxidation of the gas at reduced temperatures and at concentrations below the LEL. In its simplest embodiment, a Pt coil serves as catalyst, heater and thermometer. ${ }^{4,5}$ By passing an electric current through the coil, it is heated to a temperature sufficient for the Pt surface to catalytically oxidize the combustible mixture; the heat of oxidation is measured as a resistance variation in the Pt wire.

The pellistor is by far the most widely used electro-catalytic gas detector. ${ }^{6}$ It is a major improvement on the Pt coil and has all but supplanted that simple design. Patented by Baker ${ }^{7}$ in 1962 , the pellistor consists of a bead of low-porosity alumina encapsulating a platinum wire; the alumina bead is impregnated with suitable catalysts. Here again the Pt serves as a resistance thermometer and the catalyst heater, but is no longer used as the catalyst itself. More active catalysts, such as palladium, oxides of palladium, iridium and thorium are chosen instead for their superior ability to catalyze flammable gases. ${ }^{8}$ With better catalysts, device temperatures needed for catalysis drop, lowering power consumption and reducing wear of the Pt resistance thermometer. The high surface area of the alumina support lends a correspondingly high surface area to the catalyst improving device response and poisoning resistance.

\subsection{Micromachining approaches}

Pellistors have several disadvantages, including slow response time, high power consumption, and large size (Table 1). Often, hand manufacture and hand sorting of pellistors is required. ${ }^{9}$ Batch fabrication, miniaturization, flexible, precise

${ }^{\dagger}$ Currently employed by General Motors. 


\section{DISCLAMIER}

Portions of this document may be illegible in electronic image products. Images are produced from the best available original document. 
control of sensor geometry and the ability to choose from a wide variety of basic building materials make integrated circuit (IC) manufacturing and micromachining a potential solution to many of the problems of conventionally manufactured sensors. ${ }^{10}$ Most efforts to miniaturize calorimetric gas sensors have focused on the use of micromachined dielectric membranes as platforms upon which a sensor pair is fabricated. By placing heating resistors on a thin, free-standing, dielectric membrane, high temperatures can be rapidly achieved with low electrical input power. Microhotplates, as these devices have come to be known, are capable of reaching operating temperatures of $400-500^{\circ} \mathrm{C}$ in $1-20$ msec with the application of $50-100 \mathrm{~mW} .11,12,1314,15,16,17,18$ To deposit catalytic films, thick film techniques, ${ }^{19}$ conventional sputtering and evaporation, ${ }^{16}$ sputtering through shadow masks and deposition from catalyst-impregnated slurries, ${ }^{12}$ and selective-area $\mathrm{CVD}^{13,20,21}$ have been used.

\begin{tabular}{|c|c|c|c|}
\hline & Pellistor $^{\dagger}$ & Hotplate $^{*}$ & Microbridge $^{\ddagger}$ \\
\hline Size & $\sim 1 \mathrm{~mm}^{3}$ & $\sim 1 \mathrm{~mm}^{2}$ & $100 \times 10 \times 2 \mu \mathrm{m}$ \\
\hline Power $[\mathrm{mW}]$ & $250-450$ & 100 & 35 \\
\hline $\begin{array}{l}\text { Thermal sensitivity } \\
{\left[{ }^{\circ} \mathrm{C} / \mathrm{mW}\right]}\end{array}$ & $1-2$ & 4 & 16 \\
\hline Response time ${ }^{*}$ & $15 \mathrm{sec}$ & $20 \mathrm{msec}$ & $0.2 \mathrm{msec}$ \\
\hline Gas sensitivity & $<100 \mathrm{ppm} \mathrm{H}_{2}$ in air & $1000 \mathrm{ppm} \mathrm{H}_{2}$ in air & $100 \mathrm{ppm}_{2}$ in air \\
\hline
\end{tabular}

Table 1: Comparison of a commercially available pellistor and micromachined versions. $\dagger_{\text {City }}$ Technology Limited $4 P$; ${ }^{*}$ Reference 12; IReference $21 .{ }^{r_{\text {Ratio }}}$ of $550^{\circ} \mathrm{C}$ operating temperature to the power required to achieve $550{ }^{\circ} \mathrm{C}$; ${ }^{\ddagger}$ Time to reach $90 \%$ of steady-state operating temperature.

Another approach to miniaturization of calorimetric gas sensors is the use of free-standing micromachined filaments, or microbridges. ${ }^{22}$ It is common to deposit and pattern a thin film of platinum on a glass substrate and then etch the glass to produce a free-standing bridge; only the ends of the bridge remain attached to the un-etched portions of the substrate. A calorimetric gas sensor based on a polysilicon microbridge platform was recently reported ${ }^{21}$ and is the object of electrothermal modeling presented in this paper. Similar microbridges have been explored as potential incandescent light sources and hot-wire anemometers, ${ }^{23,24}$ but have yet to be fully exploited for chemical sensing. Here, a free-standing polycrystalline silicon bridge is used as the catalyst heater and as the resistance thermometer (Fig. 1). A typical bridge is $10 \mu \mathrm{m}$ wide, $2 \mu \mathrm{m}$ thick and is elevated above the substrate by a $2 \mu \mathrm{m}$ air gap; lengths range from $100 \mu \mathrm{m}$ to $1 \mathrm{~mm}$. A thin, $0.25 \mu \mathrm{m}$ PECVD silicon nitride film envelopes the polysilicon and passivates it against oxidation at operating temperatures. A high-surfacearea catalyst is deposited on the encapsulated bridge by a special, area-selective, " $\mu$-CVD" process described elsewhere. ${ }^{21}$
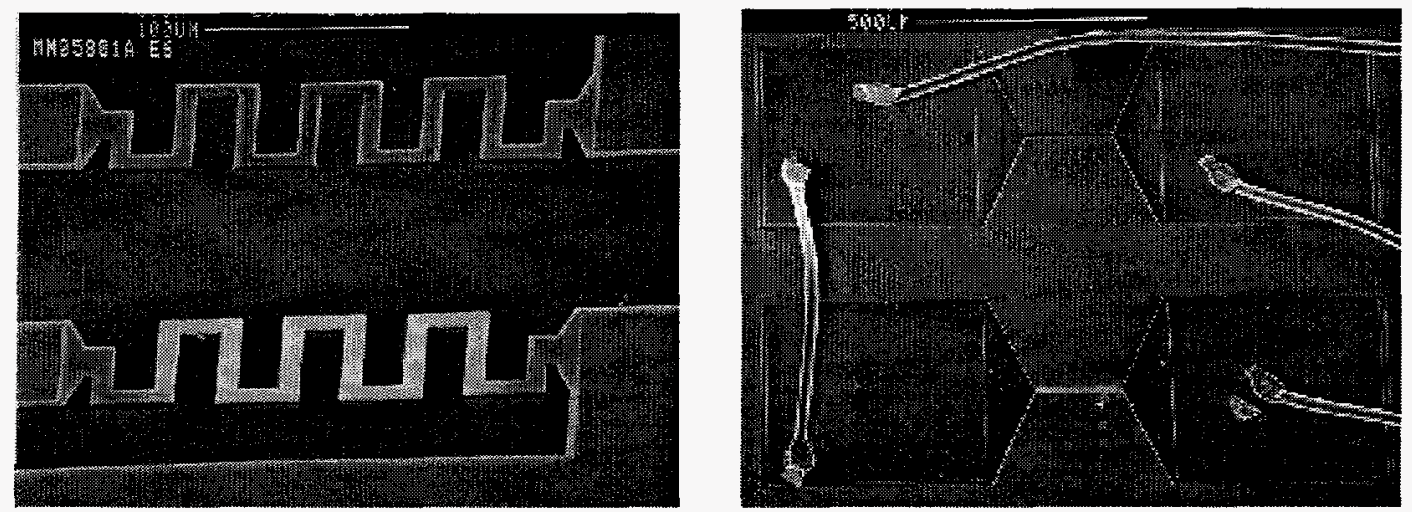

Figure 1: Left: A Scanning Electron Micrograph (SEM) plan view of two meandered polysilicon microbridges. The lower bridge was selectively coated with a thin $(\sim 0.1 \mu \mathrm{m})$ layer of $P t$ using $\mu-C V D$. In a differential gas sensing mode, the upper, uncoated, bridge serves as the inactive element, while the lower is the active device. Right: Two straight microbridges. The lower has a thick $(\sim 1 \mu m)$ layer of Pt.

A comparison among the various important characteristics of pellistors, $\mu$-hotplate and $\mu$-bridge gas sensors is given in Table 1. Micromachined sensors have improved thermal sensitivity and time response, reduced size and lower power consumption than the conventional device. These gains can be attributed to the miniaturization and design flexibility that IC manufacturing lends to micromachining. ${ }^{10}$ Furthermore, IC batch manufacturing is a significant improvement over the hand manufacture and sorting required for pellistors. Still, the ability of micromachined sensors to measure low concentration (ppb) of combustibles has yet to be demonstrated..$^{12,16}$ 


\subsection{SPICE modeling of a microbridge gas sensor}

The un-optimized sensing characteristics of the microbridge gas sensor are quite good (Table 1). To improve the response of this sensor and predict the thermal effects of catalyst deposition, extensive electro-thermal modeling efforts were undertaken. Using electrical analogues to thermal parameters (see reference 25 for an excellent discussion of these analogues), the differential equations of heat transfer were numerically solved with the circuit simulator PSPICE. ${ }^{\mathbf{T M}}$ This approach has several advantages over other numerical techniques and analytical methods. Whereas it is easy to insert the full-temperature dependence of thermal parameters into SPICE, it is not possible to obtain closed-form analytic solutions with the same temperature dependencies. It will be shown that the non-linear temperature dependence of thermal parameters has important implications for device response. Accurate prediction of the end temperature of a microbridge is also difficult analytically, but easily undertaken in SPICE. IR temperature measurements of electrically-heated microbridges will be presented which demonstrate the importance of end heating on device response. The pitfalls of such measurements will also be briefly summarized. Temperature measurements, current-voltage characteristics and transient response analysis are used to validate the SPICE model.

Electro-thermal modeling in SPICE usually includes the effects of conduction, convection, radiation and Joule heating. ${ }^{26}$ For the devices presented here radiation and convection losses are negligible. It is also possible to add chemical power to the model and predict the behavior of a microbridge gas sensor. Specifically, improvements in the mass-transport limited response of a microbridge gas sensor to methane are predicted; by modifying bridge geometry, the heating efficiency and chemical sensitivity are improved.

The principal advantage of modeling in SPICE compared with other numerical methods is the ability to simultaneously simulate the electro-thermal behavior of a device and its control/sense electronics. A novel constantresistance control circuit will be presented and its response when coupled to a microbridge is simulated and compared with several experiments. For instance, in situ methods of detecting catalyst deposition during $\mu$-CVD are discussed and modeled. The coupled electronic/microbridge model is required for this simulation and can be used to predict catalyst thickness as deposited.

\section{THE BASIC MICROBRIDGE ELECTRO-THERMAL MODEL IN SPICE}

The basic electro-thermal model of a microbridge is based on the work of Mastrangelo ${ }^{26}$ where the temperature of an element of the bridge is determined by Joule heat gain, and losses due to (1) conduction along the bridge, (2) conduction through the air from the hot bridge to the underlying substrate, (3) convection, and (4) radiation. The length of each element is $L / N$ for a bridge of length $L$ divided into $N$ elements. Using electrical analogues of thermal parameters, electrical components representing the various heat loss mechanisms and Joule heating gains can be implemented. For instance, heat loss by convection is modeled with a current source. Specifically, a voltage-controlled current source (VCCS) is used to make the amount of convective loss in a given element dependent on the temperature (voltage) of the node (Fig. 2).

VCCSs representing convection and radiation were included in the SPICE model for completeness, though neither mechanism was found to appreciably alter the temperature distribution of the devices presented here for their normal range of operation $\left(<800^{\circ} \mathrm{C}\right)$. The fact that the fourth-order temperature dependence of total radiation loss can be implemented in SPICE illustrates an important point. That is, non-linear variation of heat-loss mechanisms are permitted in SPICE. In contrast, closed-form analytic solutions allow for linearly varying properties at best. Furthermore, since material parameters such as electrical resistivity, thermal conductivity and heat capacity can vary in a non-linear fashion from room temperature to $800^{\circ} \mathrm{C}$, the ability to include non-linear temperature dependencies in SPICE is very important for this application. These observations are illustrated in Fig. 2 where the temperature distribution along the length of a microbridge is plotted for various temperature dependencies of key thermal parameters. The parameters referred to there are the electrical resistivity and thermal conductivity of polysilicon, $\rho$ and $\kappa$, respectively, while $\kappa_{\mathrm{g}}$ is the thermal conductivity of the ambient air.

The manner in which temperature-varying thermal conduction along the bridge was implemented deserves discussion. In PSPICETM it is possible to directly input a temperature-varying resistor, but the temperature used is a global parameter, typically chosen to be that of the ambient. What is required here is a resistor model that varies with the temperature at its location. A temperature-variable thermal resistor can be modeled by a series combination of a voltage-controlled voltage source (VCVS) and an ammeter (Fig. 2). This is possible since, by Ohms Law, resistance and voltage are proportional. In SPICE an ammeter is constructed with a zero-volt independent voltage source; it doesn't change the circuit, but permits the measurement of the current flowing at its location. Heat current into a node, $I_{i}$ is measured with the ammeter, and the output voltage of the ${ }^{i t h}$ dependent source (labeled as $E T_{i}$ ) is given as 


$$
E T_{i}=I_{i} \cdot\left(\frac{L}{N} \frac{1}{w z} \frac{1}{\kappa}\right),
$$

where the term in parentheses is the thermal resistance of an element of the bridge $L / N$ in length; $w$ and $z$ are the width and thickness, of the bridge. At this point, SPICE allows one to inject the functional form of the temperature dependence of thermal conductivity into $\kappa$ using an equation. In this way the resistor is made temperature dependent.
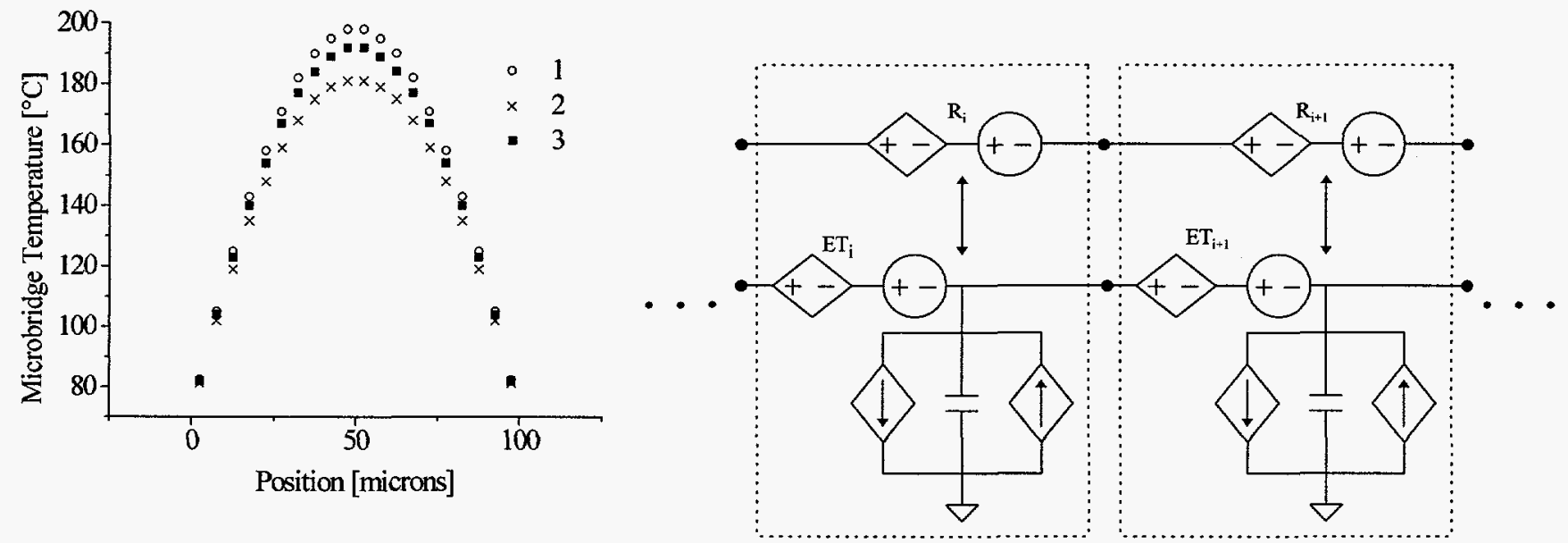

Figure 2: Left: Microbridge temperature profiles obtained with SPICE for three different conditions, and a substrate temperature of 70 ${ }^{\circ} \mathrm{C}$. Curve 1: quadratic temperature dependence of $\rho$ and $\kappa$, while $\kappa_{g}$ is constant. Curve 2: constant $\kappa$, and $\kappa_{g}$ and linear variation of $\rho$ (like the closed-form analytic solution 26). Curve 3: quadratic temperature dependencies of $\rho$ and $\kappa$, while $\kappa_{g}$ is linear. ${ }^{27}$ Symbols are explained in the text. Right: Elements ' $i$ ' and ' $i+1$ ' of the bridge in SPICE. The electrical resistors, ' $R$ ' are coupled to the thermal model at the points indicated by double arrows. The series combination of a VCVS and OV independent source functions as a temperaturdependent resistor. Joule heating (upward-pointing arrow) and losses (down arrow) are modeled with VCCSs. The heat capacity of an element is represented by a capacitor.

\section{IR TEMPERATURE MEASUREMENTS}

\subsection{Method}

To test the validity of the SPICE model, the temperature profile of a microbridge was measured using an EDO Barnes Infrascope. ${ }^{\mathrm{TM}}$ This device utilizes an InSb infrared focal plane array with a $5 \mu \mathrm{m}^{2}$ pixel size. The spectral response of the detector is $1.5-5.5 \mu \mathrm{m}$ and the IR optics have a depth of focus (DOF) of $5 \mu \mathrm{m}$. Using a two-temperature radiance technique ${ }^{28}$ the Infrascope ${ }^{\mathrm{TM}}$ first generates an emissivity map of the surface of the device under test (DUT). Then, the radiance of the powered DUT is measured and a corresponding temperature map is calculated. It is important to emphasize that because the Infrascope ${ }^{\mathrm{TM}}$ uses a staring-focal plane method, each $5 \mu \mathrm{m}^{2}$ pixel represents a separate measurement of radiance and a corresponding calculation of emissivity and temperature. However, several effects summarized next can interfere with this technique.

\subsection{Measurement errors}

The measurement system correctly calculates the emissivity and temperature of opaque materials like heavily doped silicon and polysilicon under two conditions: (1) The measured surface must not change drastically within a pixel, and (2) the measured region must be located far from bond wires. The bridge proper is only $10 \mu \mathrm{m}$ wide, but in the emissivity data appears to be $15-20 \mu \mathrm{m}$; misalignment of the bridge with respect to the pixel grid can account for most of this discrepancy (Fig. 3). A consequence of the overestimate in bridge width is the averaging of properties of the underlying substrate near the bridge, with those of the bridge itself. (Recall that the DOF is $5 \mu \mathrm{m}$ so both the substrate and surface-micromachined bridge are in focus.)

This averaging appears in both emissivity and temperature measurements for the bridge proper. The contour plot of Fig. 4 implies that the substrate surrounding the bridge is as hot as the bridge itself. It is known, however, from selective CVD of platinum on microbridges, ${ }^{21}$ that the substrate must in fact be significantly cooler than the bridge. This CVD process is thermally activated, so if both the bridge and surrounding substrate were isothermal, the deposition would be identical on 
both surfaces. On the contrary, depositions like that of Fig. 1, show that the process is highly selective to the bridge, with virtually no deposition on the substrate.

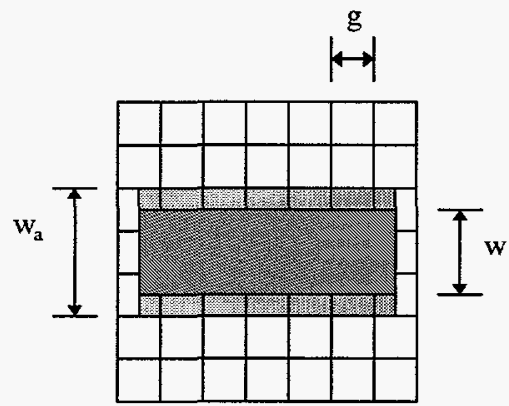

Figure 3: If a bridge of width $w=10 \mu \mathrm{m}$ is misoriented on a square pixel grid of spacing $g=5 \mu \mathrm{m}$, the apparent width $w_{a}$ is $15 \mu \mathrm{m}$.

Bond wires can diminish the emissivity of a material in its immediate vicinity relative to that of the same material located far from the bond wires. Wires elevated above the substrate are slightly out of focus because of the $5 \mu \mathrm{m}$ DOF, but still block a portion of the exitant radiation from the materials below them. Also, the detector collects emission from the bond wire to an extent determined by its height above the substrate. Hence, the emissivity in the vicinity of the bond wires is underestimated.

A material can be transparent due to good transmission properties, like silicon nitride, or simply because it is thin. Caution must be exercised where such materials are present since IR microscopes like the one discussed here implicitly assume opaque materials. To correct for the effects of transparency, the formalism of McMahon ${ }^{29}$ should be used.

Error in the measured temperature resulting from possible errors in the assumed emissivity can be estimated. For the wavelengths and temperatures of interest here, Wien's approximation ${ }^{30}$ to the Planck distribution law can be used. Based on Wien's formulation, the relative error in calculated absolute temperature due to an error in emissivity is ${ }^{31}$

$$
\frac{d T}{T}=\frac{\lambda T}{c_{2}} \cdot \frac{d \varepsilon}{\varepsilon}
$$

where $c_{2}=1.44 \times 10^{4} \mu \mathrm{m} \mathrm{K}$ and $\lambda$ is the wavelength of the emitted radiation.
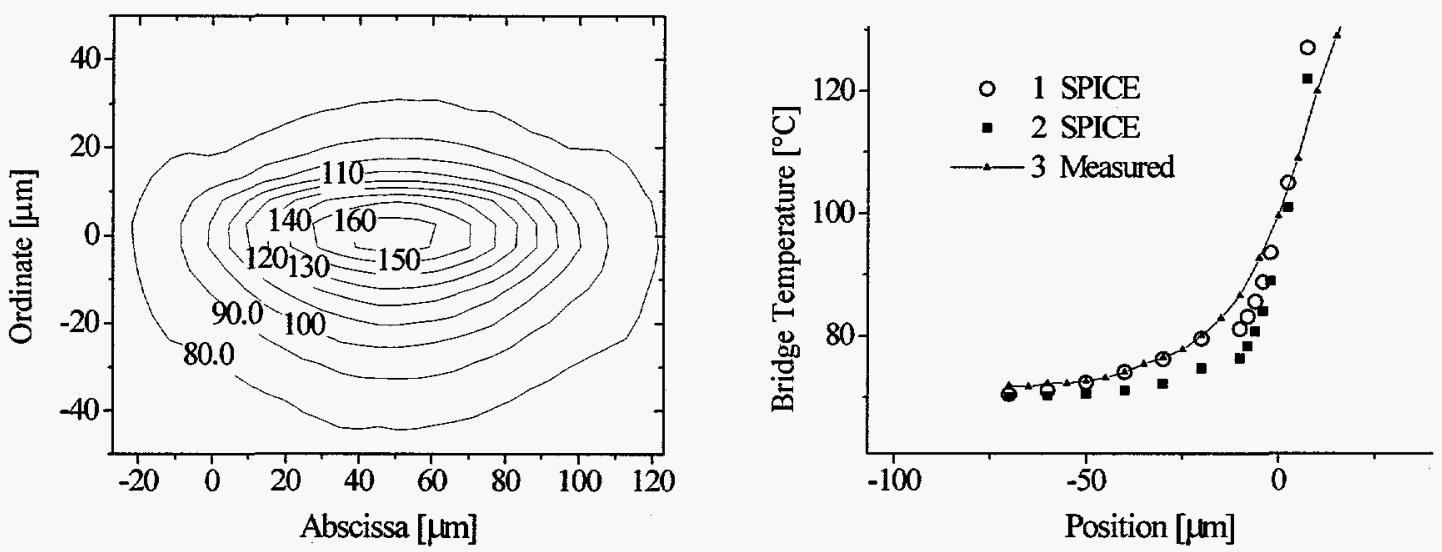

Figure 4: Left: Contour plot of temperature $\left[{ }^{\circ} \mathrm{C}\right]$ in the neighborhood of a microbridge at $28 \mathrm{~mW}$. The bridge is located along $y=0$ um between $0 \leq x \leq 100 \mathrm{\mu m}$. Right: Adding thermal resistance to the ends of the bridge mimics localized substrate heating. Curve I is from the SPICE model with $1600 \mathrm{~K} / \mathrm{W}$ at each end of the bridge. Curve 2 is SPICE result without additional resistance. Agreement between SPICE and the data, curve 3, is improved with the thermal resistance. Note that the substrate temperature is $70^{\circ} \mathrm{C}$. 


\section{BOUNDARY CONDITIONS}

\section{1 'Hard' boundary condition}

Quantitative estimates of the temperature (Fig. 4) of the bridge proper are suspect due to pixel averaging, resolution limit and the depth of focus issues described above. These issues play less of a role at the ends of a microbridge, so data obtained in these regions are expected to be reasonable. A major difference between the predicted end temperature (Fig. 2) and measured end temperature (Fig. 4) is observed. The original SPICE model assumed that the ends of the microbridge are ideal heat sinks. Measurements show, however, that significant end heating is present.

\section{2 'Soft' boundary condition in SPICE}

It is relatively easy to modify the boundary conditions in SPICE to more accurately model the temperature profile of a microbridge, especially at its ends. Elements were added to the SPICE model to represent the triangular regions at each end of the bridge (see Fig. 1); the widths of these elements were determined by the physical geometry of the triangles. Heat was allowed to diffuse through an underlying composite layer of silicon nitride and silicon dioxide to the substrate. For the measurements taken here, the substrate temperature was $70^{\circ} \mathrm{C}$. Finally, measurements indicate that the surface temperature is equal to that of the substrate for distances at least $70 \mu \mathrm{m}$ from the ends of a bridge. This boundary condition was enforced in the SPICE model. The results of these modifications are given in curve 2 of Fig. 4 . The end behavior is more accurately modeled with this 'soft' boundary condition, but still underestimates end temperatures by as much as $10^{\circ} \mathrm{C}$.

\subsection{Thermal spreading resistance}

The SPICE model described thus far has assumed that the temperature of the silicon substrate below the device remains constant, regardless of device temperature. In fact, substrate temperature in the vicinity of the microbridge increases with bridge temperature since it is not an ideal heat sink. This effect is greatest at the microbridge ends, as most of the heat generated within the device is conducted along its length and deposited in the extremities. To obtain a maximum bridge temperature of $212^{\circ} \mathrm{C}$ in a $100 \mu \mathrm{m}$ long bridge, for example, $28 \mathrm{~mW}$ of applied power is required; $21 \mathrm{~mW}$, or $77 \%$ of this is conducted outward from the center of the device and deposited at the ends, while only $7 \mathrm{~mW}$ is lost to air conduction. This leads to the observed localized substrate heating at the bridge ends.

Localized heating leads to increased thermal resistance to heat flow from the hot device to the substrate at the ends. This effect can be simply modeled in SPICE using electrical resistors. For example, a resistance of $1600 \Omega$ was placed at each end of the microbridge thermal model between the VCCSs representing conduction through oxide/nitride layers and the substrate. This equivalent, additional $1600 \mathrm{~K} / \mathrm{W}$ of thermal resistance forces the SPICE and measured end temperatures to be coincident for most of the extremities (curves 1 and 3 of Fig. 4). If one assumes that the heat conducted from the center of the bridge in steady state is deposited in a circular region of radius $a$ at the bridge ends, the thermal resistance, $R$, to heat flow into the half-space below the region is ${ }^{32}$

$$
R=\frac{1}{2 \pi \kappa a}
$$

Here, $\kappa$ is the thermal conductivity of the half-space material, which, for the substrates used here $100 \mathrm{~W}\left(\mathrm{M}^{\circ} \mathrm{C}\right)^{-1}$ is a reasonable value. ${ }^{33}$ Based on this equation, $a=1 \mu \mathrm{m}$ for $R=1600 \mathrm{~K} / \mathrm{W}$ found above. This calculation is similar to that of "spreading [electrical] resistance" encountered in electrical conduction (see reference 34 ). There, it is important to know the electrical resistance to electrical conduction into the half-space below a circle of radius $a$ over which an electrical current is flowing. By analogy, the thermal problem considered here could be called "spreading thermal resistance."

The average temperature rise within the perimeter of the circle is given by ${ }^{32}$

$$
T_{\text {ave }}=\frac{8 P}{3 \pi^{2} a \kappa}
$$

where $\mathrm{P}$ is the steady-state rate of heat input into the circular region. Continuing the example above, $\mathrm{P}$ is half the power conducted along the length of the bridge $(10.5 \mathrm{~mW})$, for which Eq. (4) gives an average localized temperature rise of $3.5^{\circ} \mathrm{C}$ in this region. This value is in agreement with the observed temperature difference between the SPICE model (lacking additional spreading thermal resistance) and the measurement shown in Fig. 4. 


\subsection{Other results}

Various results are presented in this section. First, measured current-voltage characteristics for two lengths of microbridge are compared with simulated results in Fig. 5 . At the largest voltages shown, the simulated resistance is $2 \%$ less than the measured value. Also in Fig. 5 is the variation in peak, center-point temperature of a microbridge as a function of the fractional change in resistance due to electrical heating; $\mathrm{R}_{\mathrm{o}}$ is the room-temperature resistance.

The transient response of a microbridge to a voltage pulse is easily simulated with the SPICE model and the results agree well with experiment (Fig. 6). In both cases, the current through the device is plotted as a function of the time during which it is subjected to a square voltage pulse ( $384 \mathrm{mV}$ offset, $2.56 \mathrm{~V}$ peak, $5 \mathrm{msec}$ duration, $10 \mathrm{~Hz}$ ). With reference to Fig. 6 the response can be described as follows: (A) On the initial rise of the pulse, the current through the bridge is at its highest since it is still relatively cool. Then the current decays to a steady-state value (B) as the device heats to a steady-state resistance. The decay time is a function of the thermal time constant of the bridge as well as the height of the voltage pulse. (C) When the pulse returns to its offset value on its falling edge, the current is at a minimum as the resistance is still high. (D) The current then increases as the device cools; the decay time here is inversely proportional to the thermal diffusivity of the microbridge material. It should be noted that the offset voltage used herein did not appreciably heat the device. Slight differences $(2 \%)$ between the simulated and measured current response is within the tolerance of the known material parameters and measurement uncertainties.
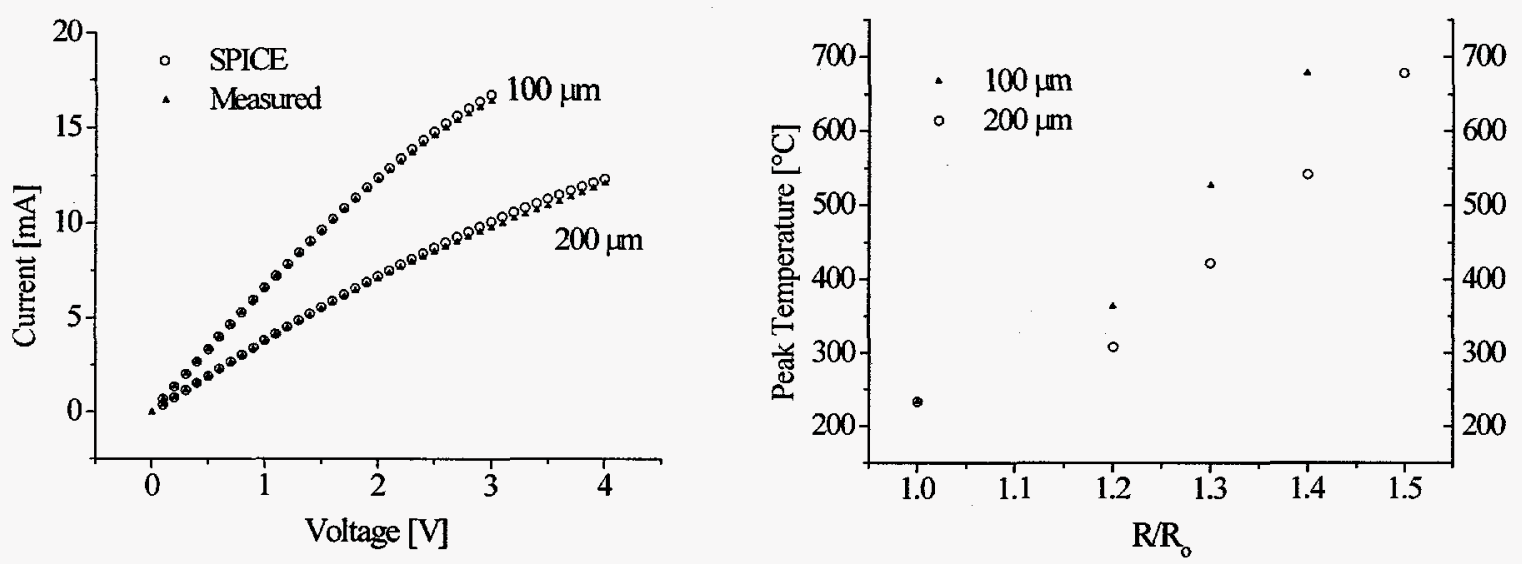

Figure 5: Left: i-v curves for 100 and $200 \mu \mathrm{m}$ bridges. Right: Center-point temperature as a function of the fractional increase in resistance over the room-temperature resistance, $R_{O}$.
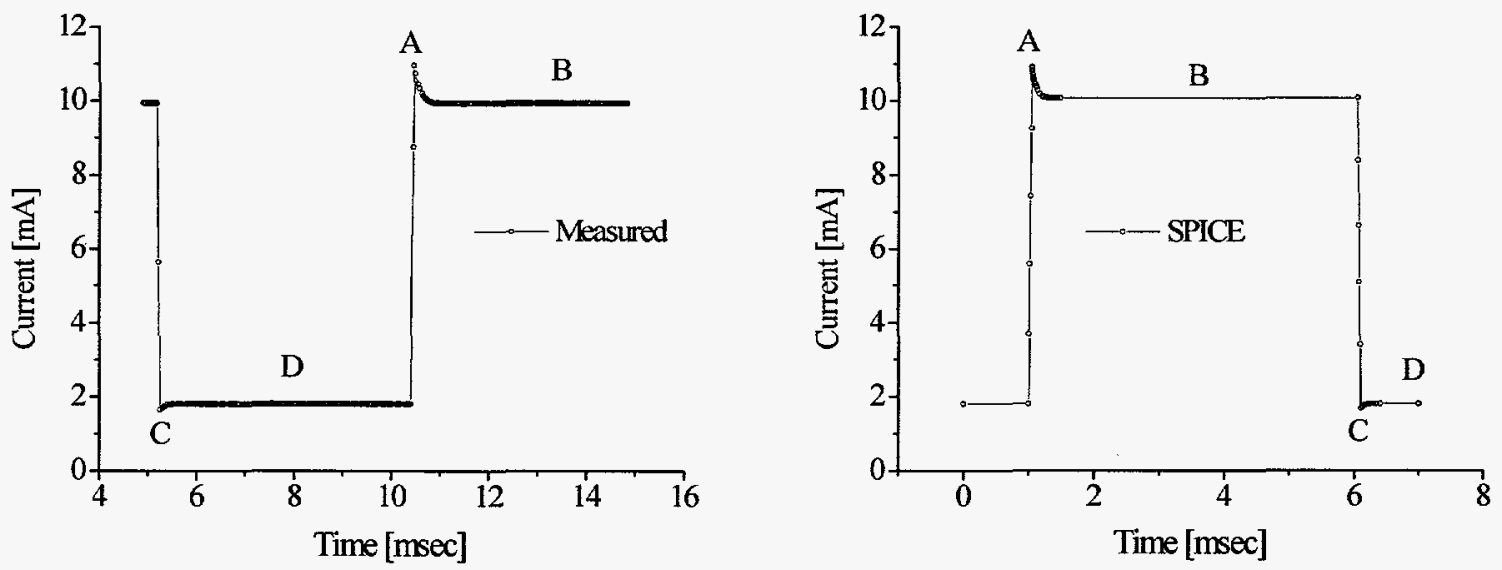

Figure 6: Simulated (left) and measured (right) response of a microbridge to a voltage pulse. The curves are displaced in time relative to one another. Alphabetic indicators are explained in the text. 


\section{COUPLED-MODEL}

5.1 Unique constant-resistance control circuit

Among the most compelling reasons for using SPICE for electro-thermal modeling is the ability to simultaneously simulate the behavior of the microbridge and its control/sensing electronics. For the microbridge gas sensor, constant resistance (or equivalently, constant temperature) operation is preferred for it reduces the likelihood of catalyst overheating and bridge fusing. Also, isothermal regulation prevents catalyst temperature from varying with gas concentration. ${ }^{16}$ A novel constantresistance control circuit (Fig. 7) was used here. The electro-thermal SPICE model of the microbridge presented above was coupled with the SPICE model of the control circuit. Results presented henceforth were obtained from this coupled model.
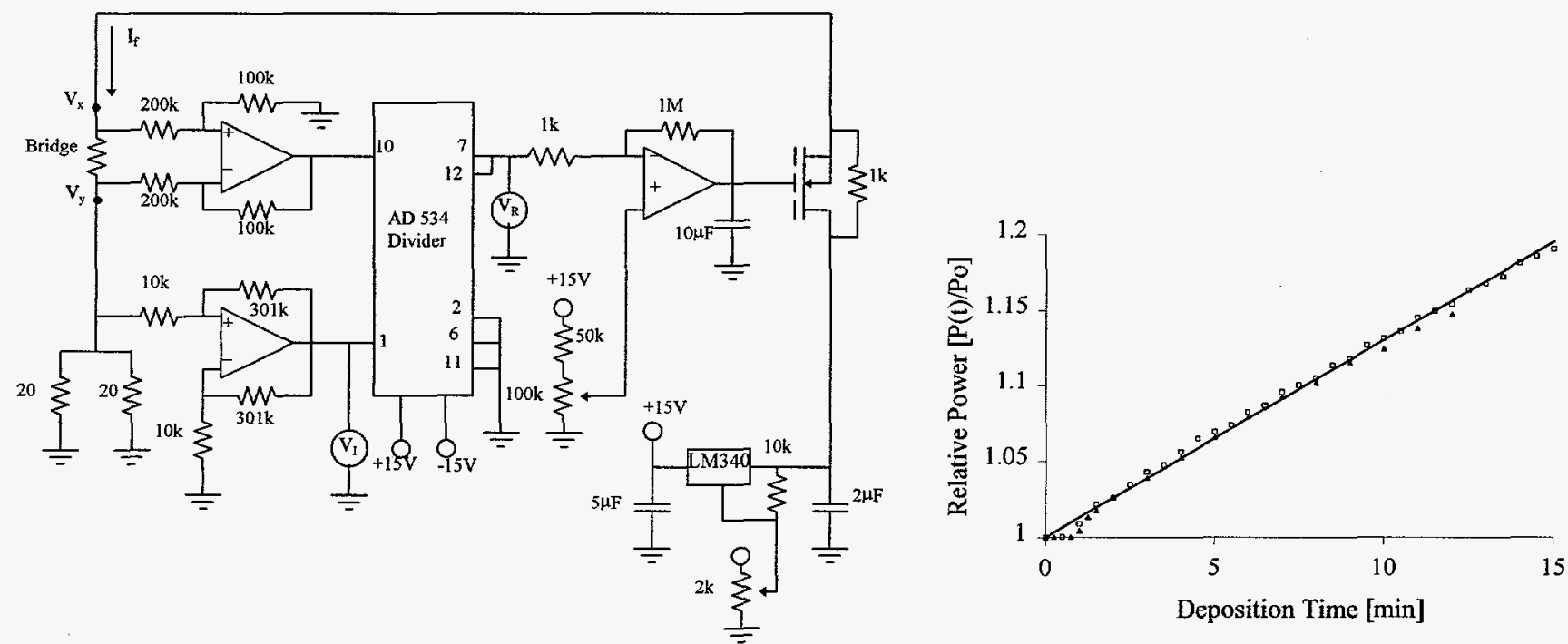

Figure 7: Left: Constant-resistance control circuit. The voltage and current of the bridge are independently monitored and input to the divider to obtain the bridge resistance. This is compared with a reference (either pulsed or continuous) and the power to the bridge is adjusted accordingly. Right: Power, $P(t)$, provided by the control circuit to maintain constant resistance during catalyst deposition relative to the power required without catalyst $\left(P_{0}\right) .12 \mathrm{~min}$. and $15 \mathrm{~min}$. depositions are shown along with a linear fit to the latter data.

\subsection{Deposition modeling: continuous set point}

Selected-area $\mu$-CVD is used to deposit catalytic films on microbridges to complete the fabrication of microbridge gas sensors. ${ }^{21}$ The bridge is electrically heated with the constant-resistance control circuit; its self-heating decomposes an organometallic precursor only on its surface (Fig. 1). As the thickness of the deposited catalyst increases, so does the ability of the deposited film to conduct heat along the length of the device. This means that the power $P(t)$ required to maintain the microbridge/catalyst system at a constant resistance $R$ increases with deposition time (Fig. 7). The power can be written

$$
P(t)=P_{o}+P_{c}(t)
$$

where $P_{\mathcal{C}}(t)$ is the increasing power conducted by the catalyst, and $P_{O}$ is the power required to maintain $R$ prior to deposition. Fig. 7 shows that with the exception of a brief initial nucleation time where the film is discontinuous, $P_{\mathcal{C}}$ increases linearly with time. For a fixed thermal conductivity of the catalyst, this implies a linear rate of increase in thickness, $z_{\mathcal{C}}$, of the deposited film. By the chain rule the growth rate at constant $R$ is $^{31}$

$$
\left.\frac{d z_{c}}{d t}\right|_{R}=\left.\frac{d P_{c}}{d t}\right|_{R} \cdot\left(\left.\frac{d P_{c}}{d z_{c}}\right|_{R}\right)^{-1}
$$

The first factor is obtained from the data of Fig. 7. The second can be obtained with the coupled SPICE model. The composite thermal conductivity $\mathrm{K}$ of the polysilicon microbridge with deposited catalyst is 


$$
\kappa\left(z_{c}\right)=\kappa_{p o l y}+\left[\frac{(2 z+w) z_{c}}{w z}\right] \cdot \kappa_{\mathcal{C}} \cdot f .
$$

The term in brackets is the ratio of cross-sectional area of the deposited film to that of the polysilicon bridge (the bridge has width $w$ and thickness $z$ ). The factor $f$ accounts for the fact that the deposited film is porous so its actual conductance is less than that implied by the bulk conductivity $\kappa_{c}$ of the catalyst $(f \leq 1)$. When Eq. (7) is inserted into Eq. (1) a composite thermal resistance $(\sim 1 / \kappa)$ of a bridge element is defined. This addition to the model allows for the determination of $P_{\mathcal{C}}$ as a function of $z_{\mathcal{C}}$.

From a $15 \mathrm{~min}$. deposition at $\mathrm{R}=1.4 \mathrm{R}_{\mathrm{o}}, d P_{c} / d t=0.82 \mathrm{~mW} / \mathrm{min}$. For this same set point and $f=1 / 3$, SPICE calculations yield $d P_{c} / d z_{c}=14 \mathrm{~mW} / \mu \mathrm{m}$. Combining these values using Eq. (6) a platinum growth rate of $59 \mathrm{~nm} / \mathrm{min}$ is obtained. For the sake of comparison, another deposition was performed at the same set point, but for only 12 minutes. Subsequent to deposition, this second device was cross-sectioned in four places along its length using a focused ion beam (FIB). A catalyst thickness distribution was derived from the cross-sections, and from this an average thickness of $0.67 \mu \mathrm{m}$ was obtained. In comparison, the growth rate determined above implies a thickness of $0.7 \mu \mathrm{m}$ at 12 minutes. Recall that a value of $f=1 / 3$ was used in this calculation indicating that the deposited film has a thermal conductance one third that of the bulk value. This difference is reasonable based on the porosity seen in FIB cross section, but is at this point somewhat arbitrary. Analysis of pulsed deposition monitoring provided in the next section supports this value of $f$.

\subsection{Deposition modeling: pulsed set point}

The small heat capacity of microbridges contributes to their rapid thermal response time, $0.2 \mathrm{msec}$ for the devices described here. Deposited catalyst significantly increases the heat capacity of the microbridge/catalyst system, introducing a measurable delay in the transient response of the system to a heating pulse. Pulsed in situ monitoring of catalyst growth is based on this principle. As catalyst thickness increases, so does the heating delay.

The electrical analogue of the heat capacity $[\mathrm{J} / \mathrm{K}]$ is capacitance $[\mathrm{F}=\mathrm{C} / \mathrm{V}]$. In SPICE the heat capacity of the $i^{t h}$ element is implemented as a capacitor $C_{i}$ placed between the node and ground. The value of the capacitor is determined by the specific heat of the material, $c\left[\mathrm{Jm}^{-3} \mathrm{~K}^{-1}\right]$, and the volume of the element. Prior to deposition only the heat capacity of the polysilicon bridge is required. During, or subsequent to deposition, another capacitor must be placed in parallel with the bridge capacitance to represent the heat capacity of the catalyst.

The response of the microbridge/catalyst system to a heating pulse was simulated and compared with experiment. In both cases, a square reference voltage was applied to the non-inverting input of the difference amplifier in the control circuit. Good agreement is observed: Heating delay due to deposited catalyst, and an increase in steady-state power required to maintain peak resistance are present in simulation and data alike. The latter effect is the same as that already discussed in the previous section, that is, the deposited catalyst conducts $P_{\mathcal{C}}$ Joules of heat per second along the length of the device. Note that the simulated and experimental values of $P_{c}$ are within $10 \%$ of one another. Also note that the analysis of the previous section for continuous monitoring can be used with steady-state delay data of the pulsed method, since they are both based on the same effect.

The two curves shown in each graph of Fig. 8 represent the transient response (1) before, and (2) after deposition at $100 \%$ duty cycle for 12 minutes at $1.4 \mathrm{R}_{\mathrm{o}}$. This was the same deposition used to evaluate the continuous in situ monitoring technique of the previous section. One would expect, then, that the value of $f$ obtained here would correspond closely to that of the continuous technique. In the simulation of Fig. 8 values of $f=1 / 3$ and $z_{\mathcal{C}}=0.7$ micron were used for comparison with the continuous technique. The resulting heating delay (measured at $40 \mathrm{~mW}$ ) was $0.29 \mathrm{msec}$ as compared with the measured value of $0.35 \mathrm{msec}$; this represents a $15 \%$ error. Using $f=0.37$, however, yields the correct heating delay of $0.35 \mathrm{msec}$. This value of $f$ is within $10 \%$ of that used in the continuous set-point simulation.

\subsection{Chemical response modeling}

Oxidation of a combustible species on the catalyst surface adds heat to the microbridge/catalyst system. The heat added per unit time in a particular sensor response regime, $P_{\text {regime }}$, due to this chemical reaction can be included in the SPICE model. Since it is a heat flow, $P_{\text {regime }}$ at a given node is generally modeled as a voltage-controlled current source (VCCS) whose magnitude is proportional to the temperature of the node. 

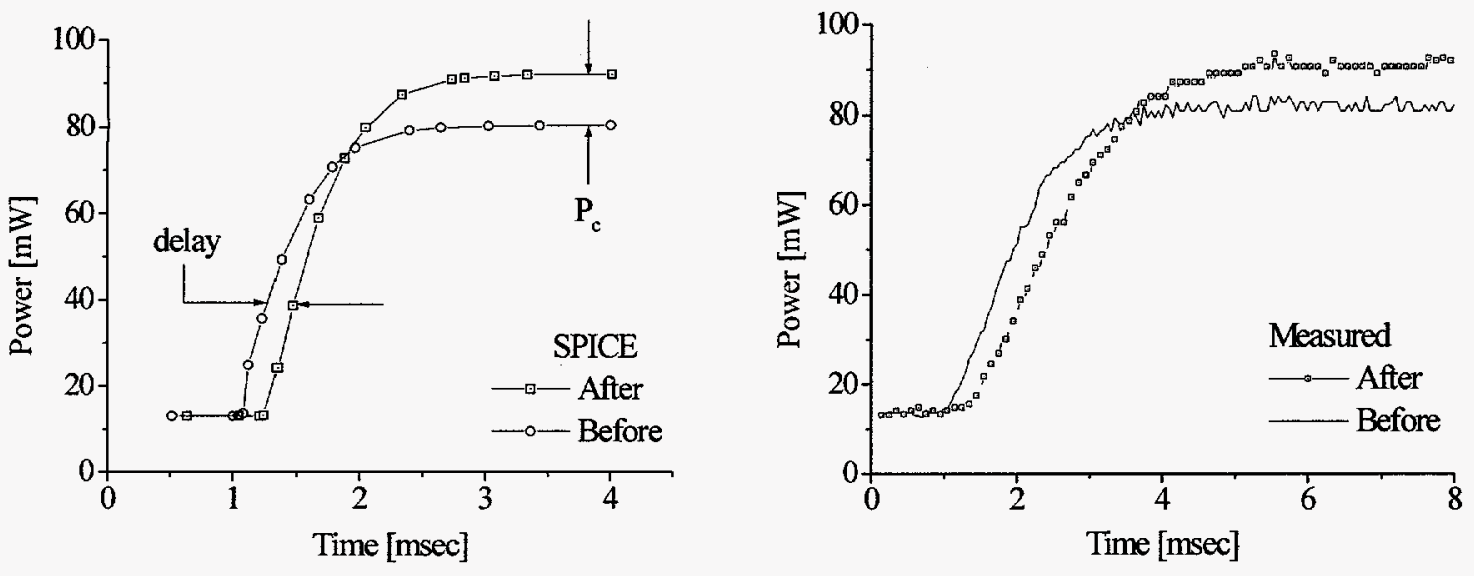

Figure 8: The response of a $100 \mathrm{um}$ bridge to a $1.3 R_{0}$ heating pulse; the power is that required to maintain this set point. The pulse is delayed after deposition with respect to that before. Left: SPICE simulation for $f=1 / 3$ and $z_{\mathcal{C}}=0.7$ micron. Right: experiment.

Electro-catalytic gas sensors exhibit a distinct threshold temperature, $T_{t}$ above which device response is relatively constant with increasing temperature. For $T \geq T_{t}$ device response is limited by mass-transport of the gas species to the catalyst surface (MTL). For a platinum catalyst, the threshold temperature for methane $\left(\mathrm{CH}_{4}\right)$ is $400{ }^{\circ} \mathrm{C} .{ }^{35}$ Below this threshold, device response is exponentially suppressed (reaction-rate limited or RRL), while above it, it is relatively constant (MTL). Chemical input power can then be modeled as

$$
\begin{array}{ll}
P_{M T L}=\gamma A H D[C] & \text { for } \quad T \geq T_{t} \\
P_{R R L} \approx 0 & \text { for } T<T_{t}
\end{array} .
$$

Here, $\mathrm{A}$ is the geometrical area of the device, $\mathrm{D}$ is the diffusion constant for transport of the combustible species to the catalyst, $\mathrm{H}$ is the enthalpy of reaction and $[\mathrm{C}]$ is the concentration of the gas. The exact value of the constant $\gamma$ is not required for the following discussion since the relative response of the detector to changes in its geometry will be presented.
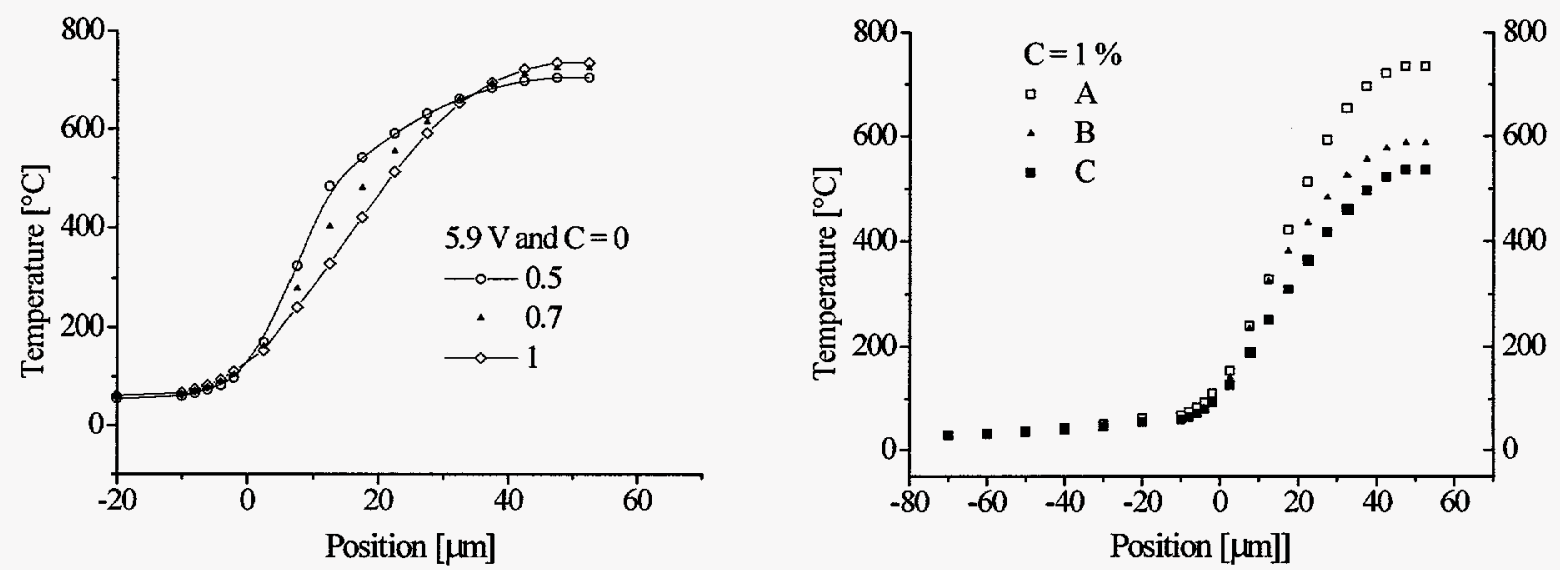

Figure 9: SPICE results. Left: Change in temperature with fractional reduction in width, '1' is the original width and '0.5,' is half that. Right: Change in temperature for three conditions explained in Table 2 and the text. $C$ is the concentration of methane in air. Since the temperature distribution is symmetric about the midpoint of a bridge, only half the profile for a 100 pm bridge is shown here.

It is possible to improve the chemical response of the sensor through a simple alteration in device geometry. By reducing the cross-sectional area of the microbridge at its ends, electrical resistance and Joule heating there are increased, and end-point temperature is enhanced relative to central temperatures. This effect was modeled with SPICE; the width of 
the end of a bridge was symmetrically reduced to $(1-a)$ of its original width for $20 \%$ of the length at each end. The enhancement in end temperatures and reduction in center temperatures with $(1-a)$ is evident in Fig. 9 (left) where a drive voltage of $5.9 \mathrm{~V}$ was used for each curve, and an ambient of air was assumed.

Practical limits to the extent to which the cross-section can be reduced are set by (a) the maximum allowable current density and (b) the mechanical stiffness of the microbridge. For a $100 \mu \mathrm{m}$ device, a current limit of $30 \mathrm{~mA}$ generally must be observed (for $w=10 \mu \mathrm{m}$ ) to prevent fusing. For a device temperature of $1.4 \mathrm{R}_{0}$ this implies that $(l-a) \geq 0.7$. Beam-bending analysis shows that $(I-a)=0.7$ for $20 \%$ of each end of a bridge does not significantly increase the likelihood that a $100 \mu \mathrm{m}$ bridge will be deflected to the substrate during release-etch drying. Only a $3 \%$ reduction in the applied force necessary for stiction is incurred when $(1-a)$ is reduced from one to 0.7 .

The right-hand graph of Fig. 9 demonstrates how bridge temperature is altered for three conditions explained in Table 2. The concentration, $\mathrm{C}$, referred to there is for methane in air with a threshold temperature of $400^{\circ} \mathrm{C}$ assumed.

\begin{tabular}{|c|c|c|c|c|}
\hline Condition & Bridge Width & $\mathbf{R} / \mathbf{R}_{\mathbf{o}}$ & Power [mW] & Response Increase [\%] \\
\hline A & $\mathrm{w}$ & 1.4 & 141 & 0 \\
\hline $\mathrm{B}$ & $0.7 \mathrm{w}$ & 1.4 & 108 & 31 \\
\hline $\mathrm{C}$ & $\mathrm{w}$ & 1.27 & 108 & 20 \\
\hline
\end{tabular}

Table 2: Conditions of Fig. 9 . "Response" is to $1 \%$ methane in air. Response increase is relative to condition ' $A$ '.

Table 2 and Fig. 9 (right) explain that the reduction in geometry improves device response in two ways. (1) Localized end heating above the threshold temperature of reaction permits the addition of chemical energy there. (2) The baseline power required to maintain the set-point resistance is decreased, and the signal-to-noise ratio is accordingly enhanced. Comparison of conditions A and B illustrates these points. It is interesting to note that simply reducing the power provided to a device with the original geometry (condition $\mathrm{C}$ ) improves the response. Here again, device power is reduced and signal-to noise is enhanced. Still, a $30 \%$ reduction in the end width (B) yields a $11 \%$ improvement over that of $\mathrm{C}$ since a larger portion of the bridge is above the threshold temperature. It is important to bear in mind that these results are for methane. Enhancements in sensor response and efficiency will be even greater for gases such as ethylene, ethane and hydrogen whose threshold temperatures are smaller than methane. This analysis represents a lower limit to the expected improvements in response for these gases.

\section{CONCLUSIONS}

The heat-transfer equations for an electrically-heated microbridge were easily solved in SPICE even when important nonlinearities in heat loss mechanisms and thermal parameters were included. IR temperature measurements indicate that heating at the ends of a microbridge is significant. This effect was modeled in SPICE with a modification in end geometry and the inclusion of thermal spreading resistance. The major advantage of SPICE over other numerical methods is its ability to iteratively solve the coupled system consisting of the electro-thermal model of the microbridge gas sensor and its unique constant-resistance control circuit. With this coupled model, in situ deposition monitoring techniques were accurately simulated. The chemical response of the sensor was also predicted. Simulations show that reducing the end geometry of a microbridge provides an easy means of improving chemical sensitivity and heating efficiency without significantly sacrificing the mechanical or electrical integrity of the device.

\section{ACKNOWLEDGMENT}

Sandia is a multiprogram laboratory operated by Sandia Corporation, a Lockheed Martin Company, for the United States Department of Energy under contract DE-AC04-94AL85000. The microbridges shown here were produced at Sandia's Microelectronics Development Laboratory (MDL); the excellent technical assistance of the engineers and operators of the MDL is gratefully acknowledged. Special thanks to Patrick Shea for the SEMs shown herein, and Alan W. Staton for assistance with catalyst depositions. Finally, thanks to Maria for her encouragement and support.

\section{REFERENCES}

1 J.G. Firth, A. Jones, T.A. Jones, "The principles of the detection of flammable atmospheres by catalytic devices", Combustion and Flame, 21 (1973) 303-311

2 D. Balfour, "The developing role of gas detection", Control and Instrumentation, 24 (1993) 19-21. 
3 M.G. Jones and T.G. Nevell, "The detection of hydrogen using catalytic flammable gas sensors", Sensors and Actuators, 16 (1989) $215-$ 224.

4 J.J. Eherhardt, L. Colin, A. Accorsi, M. Kazmierczak and I. Zdanevitch, "Catalytic oxidation of methane on platinum thin film", Sensors and Actuators B, 7 (1992) 656-660.

5 M.G. Jones and T.G. Nevell, "The detection of hydrogen using catalytic flammable gas sensors", Sensors and Actuators, 16 (1989) 215224.

6 M.G. Jones and T.G. Nevell, "The detection of hydrogen using catalytic flammable gas sensors", Sensors and Actuators, 16 (1989) 215224.

7 A.R.Baker, Combustible gas-detecting, electrically heatable element, U.K. Patent 892530 (1962).

8 J .G. Firth and H.B. Holland, "Catalytic oxidation of methane over noble metals", Trans. Faraday Soc., 65 (1969) $1121-1127$.

9 E. Jones, "The Pellistor Catalytic Gas Sensor", Solid State Gas Sensors, P.T. Moseleyand and BC. Tofield eds., A. Higler (Bristol and Philadelphia) 1987.

10 R.P. Manginell, J.H. Smith and A.J. Ricco, "An overview of micromachined platforms for thermal sensing and gas detection", SPIE's

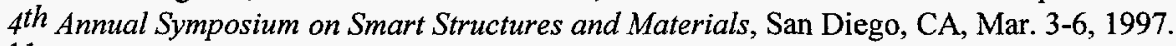

11 R.E.Cavicchi, J.S. Suehle, P. Chaparala, K.G. Kreider, M. Gaitan, and S. Semancik, "Micro-hotplate gas sensor", Tech. Digest 1994 Sol.-State Sensor and Actuator Workshop, Hilton Head, SC, 6/13-16/94, pp. 53-56.

12 M.Zanini, J.H.Visser, L.Rimai,R.E.Soltis, A.Kovalchuk,D.W.Hoffmann, E.M. Logothetis, U. Bonne, L. Brewer, O.W. Byrnum, M.A. Richard, "Fabrication and properties of a Si-based high-sensitivity microcalorimetric gas sensor", Sensors and Actuators A, (1995) 187192.

13 S. Semancik, R.E. Cavicchi, K.G. Kreider, J.S. Suehle, P. Chaparala, "Selected-area deposition of multiple active films for conductimetric microsensor arrays, 8th International Conference on Solid-State Sensors and Actuators and Eurosensors LX. Digest of Technical Papers, vol. 1, (1995) 831-834.

14 R. Aigner, F. Auerbach, P. Huber, R. Muller and G. Scheller, "Sinusoidal temperature modulation of the Si-Planar-Pellistor", Sensors and Actuators B, 18-19 (1994) 143-147.

15 R. Aigner, M. Dietl, R. Katterloher and V. Klee, "Si-planar pellistor: Designs for temperature modulated operation", 8th International Conference on Solid-State Sensors and Actuators and Eurosensors LX. Digest of Technical Papers, (1995) 839-842.

16 M. Gall, "The Si-planar-pellistor array, a detection unit for combustible gases", Sensors and Actuators B, 15-16 (1993) $260-264$.

17 M. Gall, and R. Muller, Investigation of gas mixtures with different MOS gas sensors with regard to pattern recognition, Sensors and Actuators 17 (1989) 583-586.

18 M. Gall, The Si pellistor: a low-power pellistor sensor in Si thin-film technology, Sensors and Actuators B, 4 (1991) $533-538$.

19 F. Menil, C. Lucat, H. Debeda, The thick-film route to selective gas sensors, Sensors and Actuators $B, 24-25$ (1995) 415-420.

20 S. Majoo, J.W. Schwank, J.L. Gland, and K.D. Wise, "A Selected-area CVD Method for Deposition of Sensing Films on

Monolithically Integrated Gas Detectors", IEEE Electron Device Letters, 16, 217 (1995).

21 R.P. Manginell, J.H. Smith, A.J. Ricco, D.J.Moreno, R.C Hughes, R.J. Huber, S.D. Senturia, "Selective, pulsed CVD of platinum on microfilament gas sensors," Tech. Digest 1996 Sol.-State Sensor and Actuator Workshop, Hilton Head, SC, pp. 53-56.

22 C. Vauchier, D. Charlot, G. Dalapierre and A. Accorsi, Sensors and Actuators B, 33 (1991) 33-36.

23 Y. C. Tai, R. S. Muller, and R. T. Howe, Transducers ' 85.1985 International Conference on Solid-State Sensors and Actuators.

Technical Digest of Papers, p. 445 (1985).

24 C. H. Mastrangelo and R. S. Muller, "A Constant Temperature Gas Flowmeter With A Silicon Micromachined Package", Tech. Digest 1988 Sol.-State Sensor and Actuator Workshop, Hilton Head Isl., SC, June, 1988, pp. 43 - 47.

25 S.M. Sze, Semiconductor Sensors, John Wiley and Sons, New York, 1994.

26 C.H. Mastrangelo, Thermal Applications of Microbridges, Ph.D. Dissertation, U.C. Berkeley, 1991.

27 J. Sucec, Heat Transfer, WM. C. Brown Publishers, Dubuque, Iowa, 1985, Appendix A11.

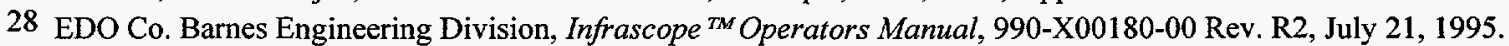

29 H.O. McMahon, Thermal radiation from partially transparent reflecting bodies, Journal of The Optical Society of America, 40 (1950) 376-380.

30 D.P. DeWitt, and G.D. Nutter, Theory and Practice of Radiation Thermometry, John Wiley and Sons, New York, 1988.

31 R.P. Manginell, A polysilicon microbridge gas sensor, Ph.D. Dissertation, The University of New Mexico, 1997.

32 H.S. Carslaw and J. C. Jaeger, Conduction of heat in solids, $2^{\text {nd }}$ Ed., Oxford University Press, 1959, pp 214-217.

33 G.A. Slack, Thermal conductivity of pure and impure silicon, silicon carbide, and diamond, J. App. Phys., 35 (1964) $3460-3466$.

34 S.M. Sze, Physics of semiconductor devices, $2^{\text {nd }}$ Ed., John Wiley \& Sons, New York, 1981, p 217.

35 J.G. Firth, Measurement of flammable gases and vapours, Detection and Measurement of Hazardous Gases, C.F. Cullis and J.G. Firth Eds., Heinemann, London, 1981.

\section{DISCLAIMER}

This report was prepared as an account of work sponsored by an agency of the United States Government. Neither the United States Government nor any agency thereol, nor any of their employees, makes any warranty, express or implied, or assumes any legal liability or responsibility for the accuracy, completeness, or usefulness of any information, apparatus, product, or process disclosed, or represents that its use would not infringe privately owned rights. Reference herein to any specific commercial product, process, or service by trade name, trademark, manufacturer, or otherwise does not necessarily constitute or imply its endorsement, recommendation, or favoring by the United States Government or any agency thereof. The views and opinions of authors expressed herein do not necessarily state or reflect those of the United States Government or any agency thereol. 